CMEARTICLE

\title{
Clinics in diagnostic imaging (193)
}

Jun Si Yuan Li $i^{1}$, MD, FRCR, Kheng Choon Lim${ }^{1}$, MD, FRCR, Winston Eng Hoe $\underline{L i m}^{1}$, MBBS, FRCR, Robert Chun Chen ${ }^{1}$, MD
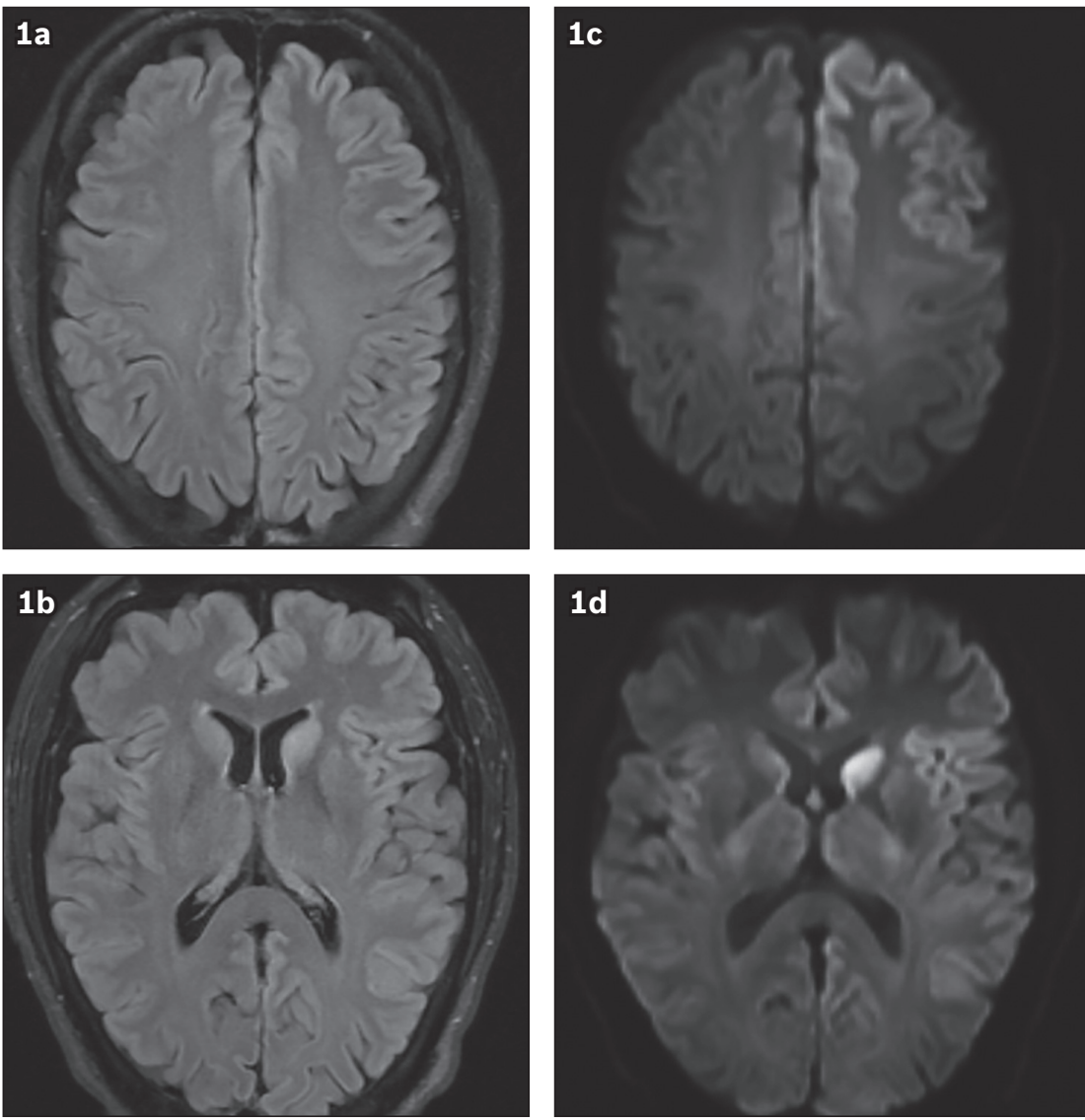

Fig. 1 ( $a$ \& b) Fluid-attenuated inversion recovery (FLAIR) and (c \& d) diffusion-weighted images.
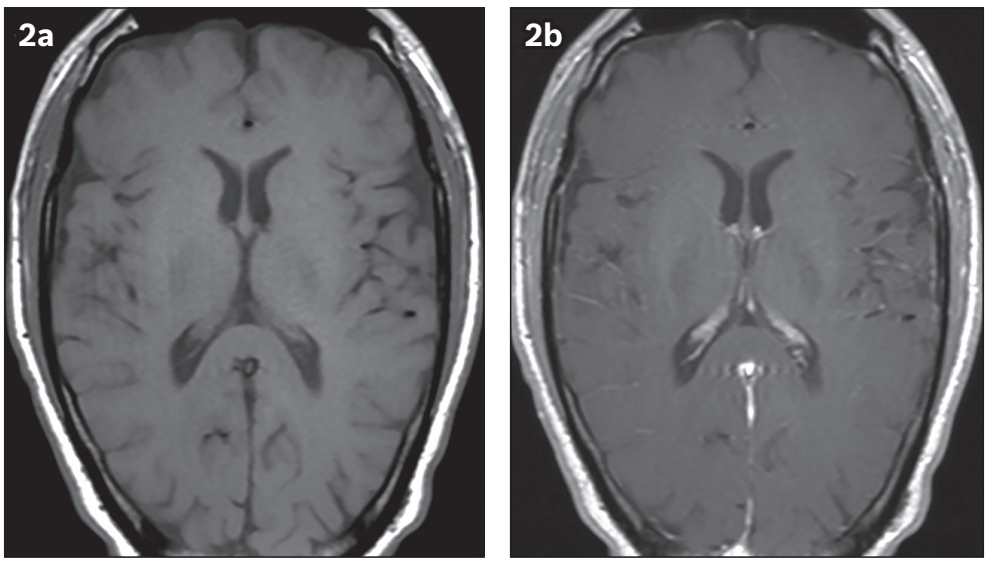

Fig. 2 (a) Pre- and (b) post-gadolinium T1-weighted images.

\section{CASE PRESENTATION}

A 68-year-old Caucasian man with no significant past medical history presented to the emergency department with a three-week history of progressive memory loss, involuntary muscular twitches deemed to represent myoclonus, and an unsteady gait. Electroencephalography (EEG) revealed periodic sharp wave complexes and cerebrospinal fluid (CSF) analysis revealed the presence of a 14-3-3 protein. What do the magnetic resonance (MR) images (Figs. 1 \& 2) show, and what is the diagnosis? 
Table I. World Health Organization diagnostic criteria for sporadic Creutzfeldt-Jakob disease.

\begin{tabular}{|c|c|c|c|}
\hline Diagnosis & Definite & Probable & Possible \\
\hline Biopsy & $\begin{array}{l}\text { Characteristic } \\
\text { neuropathology or PrPsc }\end{array}$ & - & - \\
\hline Clinical features & - & $\begin{array}{l}\text { Rapidly progressive dementia and } \geq 2 \text { of the } \\
\text { following: } \\
\text { - Myoclonus } \\
\text { - Visual impairment } \\
\text { - Cerebellar signs } \\
\text { - Pyramidal/extrapyramidal signs } \\
\text { - Akinetic mutism }\end{array}$ & $\begin{array}{l}\text { Same as 'Probable' and duration } \\
<2 \mathrm{yr}\end{array}$ \\
\hline Paraclinical tests & - & $\begin{array}{l}\geq 1 \text { of the following: } \\
\text { - EEG: periodic sharp wave complexes } \\
\text { - CSF analysis: } 14-3-3 \text { protein } \\
\text { - MR imaging: hyperintensity in the } \\
\text { caudate nucleus/putamen or } \geq 2 \text { cortical } \\
\text { regions (temporal-parietal-occipital) on either DW } \\
\text { or FLAIR sequences }\end{array}$ & $\begin{array}{l}\text { Negative or atypical result } \\
\text { from the three tests without } \\
\text { suggestion of an alternative } \\
\text { diagnosis }\end{array}$ \\
\hline
\end{tabular}

CSF: cerebrospinal fluid; DW: diffusion-weighted; FLAIR: fluid-attenuated inversion recovery; EEG: electroencephalography; MR: magnetic resonance; PrPsc: scrapie prion protein

\section{IMAGE INTERPRETATION}

Axial fluid-attenuated inversion recovery (FLAIR) images show hyperintense signal in the left caudate nucleus and asymmetric signal abnormality in the cerebral cortices, worse on the left side, primarily affecting the left frontal lobe, left insula and left temporal-parietal lobes (Figs. 1a \& b). Diffusion-weighted (DW) images demonstrate similar yet more pronounced signal abnormality in the areas mentioned above (Figs. 1c \& d). Pre- and post-gadolinium T1-weighted images show no abnormal enhancement (Figs. 2a \& b).

\section{DIAGNOSIS}

Sporadic Creutzfeldt-Jakob disease (sCJD).

\section{CLINICAL COURSE}

The patient's clinical condition progressively deteriorated over a period of 18 months; he eventually became unconscious, requiring intubation with mechanical ventilation. The course was complicated by sepsis and the patient eventually died. While no autopsy was performed, the clinical and paraclinical tests were sufficient to make a probable diagnosis of sCJD.

\section{DISCUSSION}

Creutzfeldt-Jakob disease (CJD) is a group of human prion diseases alternatively known as transmissible spongiform encephalopathies. It was first described independently by Alfons Maria Jakob and Hans Gerhard Creutzfeldt in 1920. The mechanism of prion diseases was formally proposed by Stanley Prusiner in 1982, which eventually earned him the 1997 Nobel Prize in Medicine. It is now understood that human prion diseases are caused by an alteration of the naturally existing, detergentsoluble and protease-sensitive prion protein into an abnormally folded, detergent-insoluble and protease-resistant protein, termed scrapie prion protein $\left(\mathrm{PrPsc}^{\mathrm{Sc}}\right.$ ), in the brain. ${ }^{(1)}$ The process propagates through self-replication of the newly formed $\mathrm{PrPsc}^{\mathrm{Sc}}$ that eventually results in spongiform degeneration and neuronal loss. sCJD is by far the most common and best understood subtype, accounting for about $85 \%$ of overall CJD cases, with the remaining consisting of familial (10\%-15\%) and acquired (1\%) subtypes, such as fatal familial insomnia and variant CJD, respectively. ${ }^{(2)}$ SCJD has an incidence of about one case per million people per year worldwide and peaks between the ages of 65 and 74. No gender predilection exists. ${ }^{(3)}$

The sine qua non of SCJD is rapidly progressive dementia and invariably fatal outcome; despite this, clinical symptoms can range from isolated visual disturbance, confusion and amnesia to ataxia, dementia and myoclonus. ${ }^{(3)}$ Early diagnosis of SCJD can be challenging due to the variegated signs and symptoms. To facilitate diagnosis, paraclinical tools, such as EEG, and CSF analysis can be used. EEG may show a distinctive pattern of periodic sharp wave complexes, and CSF assays can show the presence of a 14-3-3 protein. ${ }^{(3)}$ MR findings, namely high signal abnormalities in the caudate nucleus and putamen or at least two cortical regions on either DW or FLAIR sequences, have been included in the updated diagnostic criteria of SCJD. ${ }^{(4)}$ These are summarised in Table I. There have been no effective remedies to halt or slow disease progression, and treatment focuses solely on symptom alleviation. The prognosis for CJD is dismal, with patients usually typically surviving a few months to a year. sCJD has the shortest life expectancy of all the subgroups of CJD, at a median of 4-5 months upon diagnosis. ${ }^{(5)}$

Neuroimaging, in particular the DW sequence, plays an integral role in supporting the in vivo diagnosis of CJD at an early stage, due to its high sensitivity and specificity. It can give further credence to the reliability of the EEG and CSF results and, in some cases, pre-date abnormal EEG and CSF findings. ${ }^{\left({ }^{6}\right)}$ An abnormal MR image with a typical CJD pattern not uncommonly reveals an unsuspected case of CJD when the EEG and CSF findings are equivocal. Although the pathophysiology of disease 

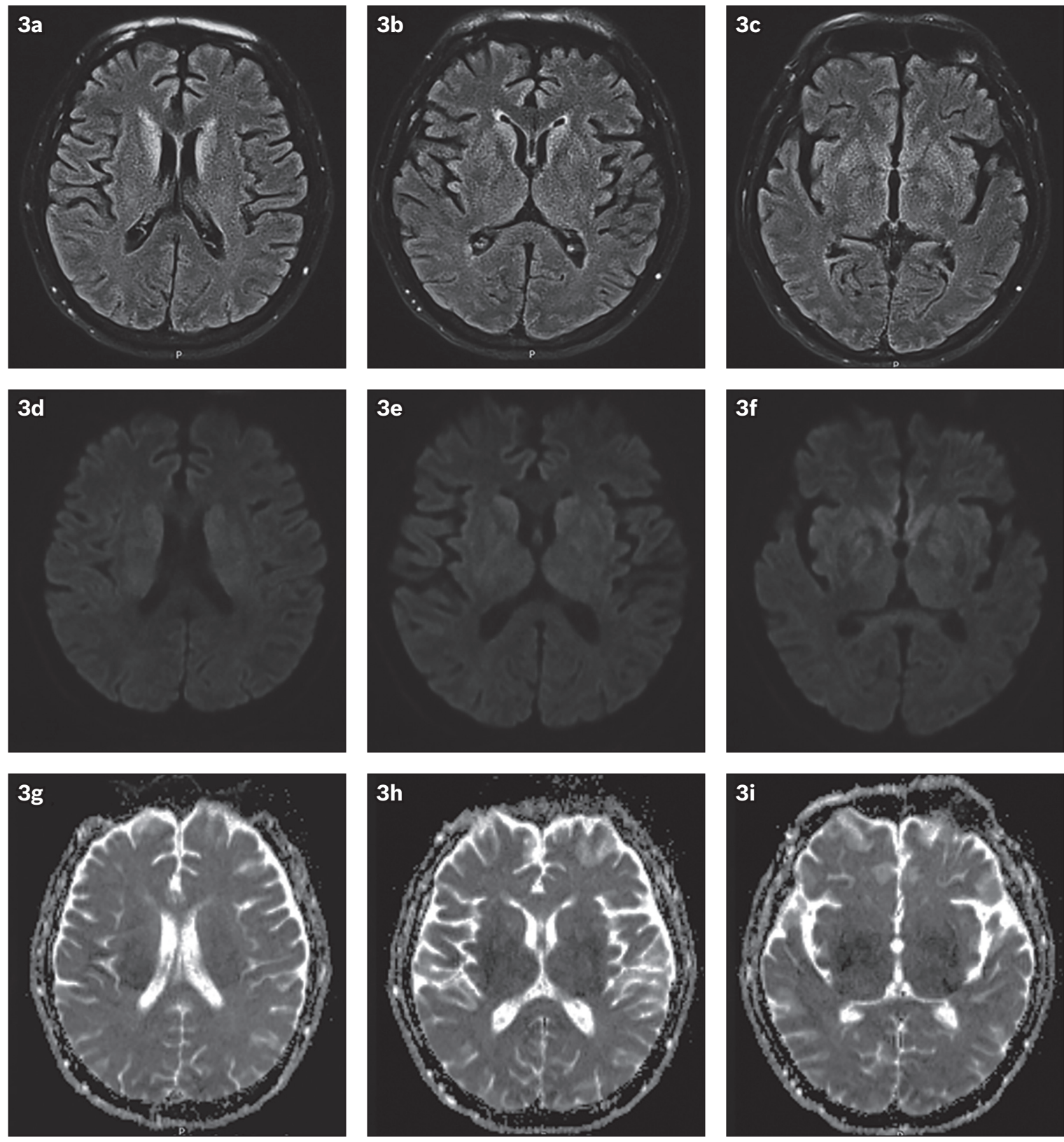

Fig. 3 Hypoxic-ischaemic encephalopathy in a 60-year-old man who presented with acute pulmonary embolism. Post-embolectomy, he developed a poor Glasgow Coma Scale score and was placed on extracorporeal membrane oxygenation. (a-c) FLAIR images show symmetrical hyperintensity in the bilateral caudate and inferior lentiform nuclei. ( $d-f)$ DW images and (g-i) apparent diffusion coefficient (ADC) map show mildly reduced diffusion in the affected areas.

manifestation on the DW sequence remains unclear, it has been postulated that restricted diffusion within the affected areas of the brain is related to underlying vacuolation or deposition of the prion protein. ${ }^{(7)}$ Three classic MR imaging patterns of sCJD on DW imaging have been reported: asymmetric involvement of the cortex and basal ganglia; involvement of the cortex only; and involvement of basal ganglia and thalami only. ${ }^{(2,8)}$ Our case demonstrates the most common pattern of CJD, involving both the cortex and basal ganglia. Although the 'pulvinar' or 'double hockey stick' signs of thalamic involvement are known to be classic radiological hallmarks attributable to variant CJD, they have also been observed in certain molecular subtypes of sCJD. (2) In addition, even when cerebellar signs may be clinically present, MR signal abnormality in the cerebellum is rarely detectable in
sCJD. Cerebellar atrophy with elevated diffusivity on apparent diffusion coefficient maps may be the only radiologic sign of cerebellar involvement. ${ }^{(2)}$ As the disease progresses, there may be an increase in both the extent and intensity of DW and FLAIR signal abnormality in the cortex and basal ganglia due to progressive spongiform degeneration. Pseudonormalisation of signal intensity is frequently observed when there is inevitable atrophy in the late stage of the disease process. ${ }^{(6)}$

Single-photon emission computed tomography and positron emission tomography/computed tomography (PET/CT) are alternative advanced neuroimaging techniques for diagnosing sCJD. PET/CT, in particular ${ }^{18} \mathrm{~F}$-FDG-PET/CT ( ${ }^{18} \mathrm{~F}$-fluoro-2-deoxyD-glucose-PET/CT), has proven useful because of its capability of detecting regions of brain tissue with altered metabolic 

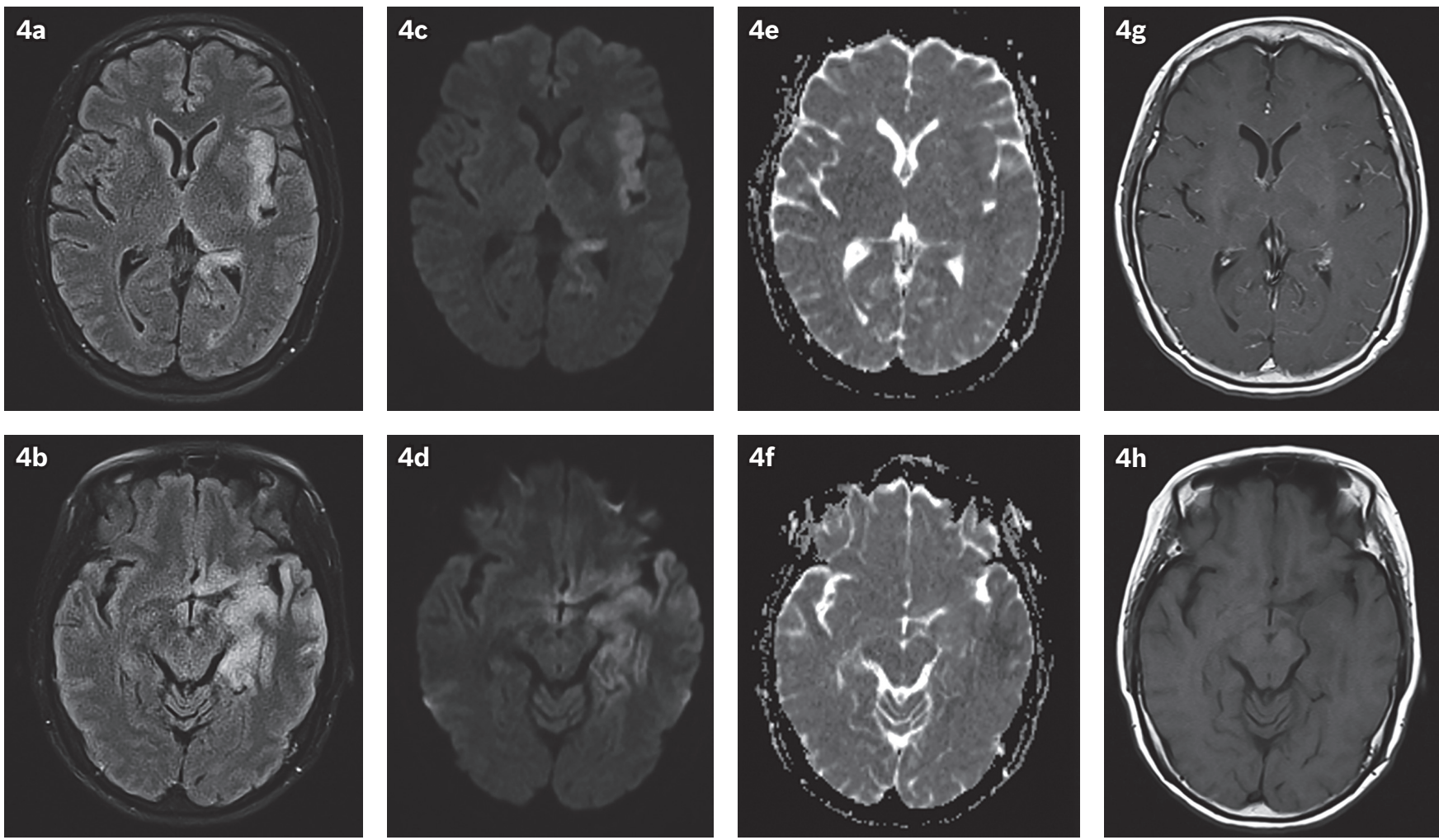

Fig. 4 Herpes simplex virus in a 66-year-old woman who presented with altered mental status, dysphagia, facial asymmetry and high fever. (a \& b) FLAIR and (c \& d) DW images show abnormal high signal and swelling in the left hippocampus, left mesial and inferior temporal lobe, left insular cortex and left basifrontal lobe, with no (e \& f) appreciable corresponding restricted diffusion in these areas on ADC map. (g \& h) Post-gadolinium T1-W images show slight leptomeningeal enhancement on the left.

status, which can pre-date abnormalities seen on conventional MR images. ${ }^{(8)}$ This promising imaging tool has not yet been universally utilised due to its lack of availability and accessibility, and therefore currently remains an effective supplement to MR imaging.

It is important to differentiate sCJD from other neurological diseases with similar clinical or radiologic patterns, because some of the latter may be treatable. We herein introduce some conditions that may mimic sCJD. In the acute setting, hypoxicischaemic encephalopathy (HIE) may demonstrate increased signal intensity in the more metabolically active regions of the brain, such as the basal ganglia, hippocampus and cerebral cortex (Fig. 3). ${ }^{(9)}$ A key differentiating factor between SCJD and HIE is the presence of any relevant history, such as a recent history of cardiorespiratory collapse. Hypoglycaemic encephalopathy shares imaging features with HIE because of its predilection to affect parts of the brain with high energy consumption. ${ }^{(10)}$ Early diagnosis is usually based on the patient's clinical history and extremely low blood glucose level $(<2.9 \mathrm{mmol} / \mathrm{L})$.

Herpes encephalitis is the most common acute fatal sporadic viral encephalitis caused by the herpes simplex virus. It can clinically manifest with an acutely decreased level of consciousness, neurological deficits, and nonspecific symptoms such as headache and fever. Unlike sCJD, herpes encephalitis (Fig. 4) typically only involves the limbic structures, with preferential involvement of the mesial temporal lobes. ${ }^{(11)}$ The basal ganglia is spared. Necrosis and haemorrhage may also occur, which has not been described with sCJD. Detection of herpes simplex virus DNA on polymerase chain reaction can confirm the diagnosis.

Posterior reversible encephalopathy syndrome is a neurotoxic state resulting from an acute change of perfusional status to the posterior portions of the brain, either due to failed autoregulation or vasoconstriction. ${ }^{(12)}$ The clinical spectrum may range from acute or subacute nonspecific symptoms of headache and altered mental status to visual disturbance and generalised seizure. Vasogenic oedema is classically seen in the posterior parietal and occipital regions, as well as the watershed regions between vascular territories (Fig. 5). ${ }^{(13)}$ Restricted diffusion is typically absent, while microhaemorrhages in the affected brain are relatively common. The key clue is the pattern of imaging abnormality and the 'reversible' course of the disorder, both clinically and radiologically, after proper treatment of the underlying causative condition.

A postictal state may manifest as transient focal T2/FLAIR and DW signal changes secondary to cytotoxic and vasogenic oedema. Involvement of the hippocampal regions (Fig. 6), splenium of the corpus callosum, cortex and subcortical white matter have been reported. ${ }^{(14)}$

Voltage-gated potassium channel encephalitis is a common subtype of autoimmune encephalitis defined by intractable epilepsy. The typical MR findings are T2 and FLAIR high signal abnormalities that almost invariably involve the limbic system; however, the cortex and cerebellum may also be involved 

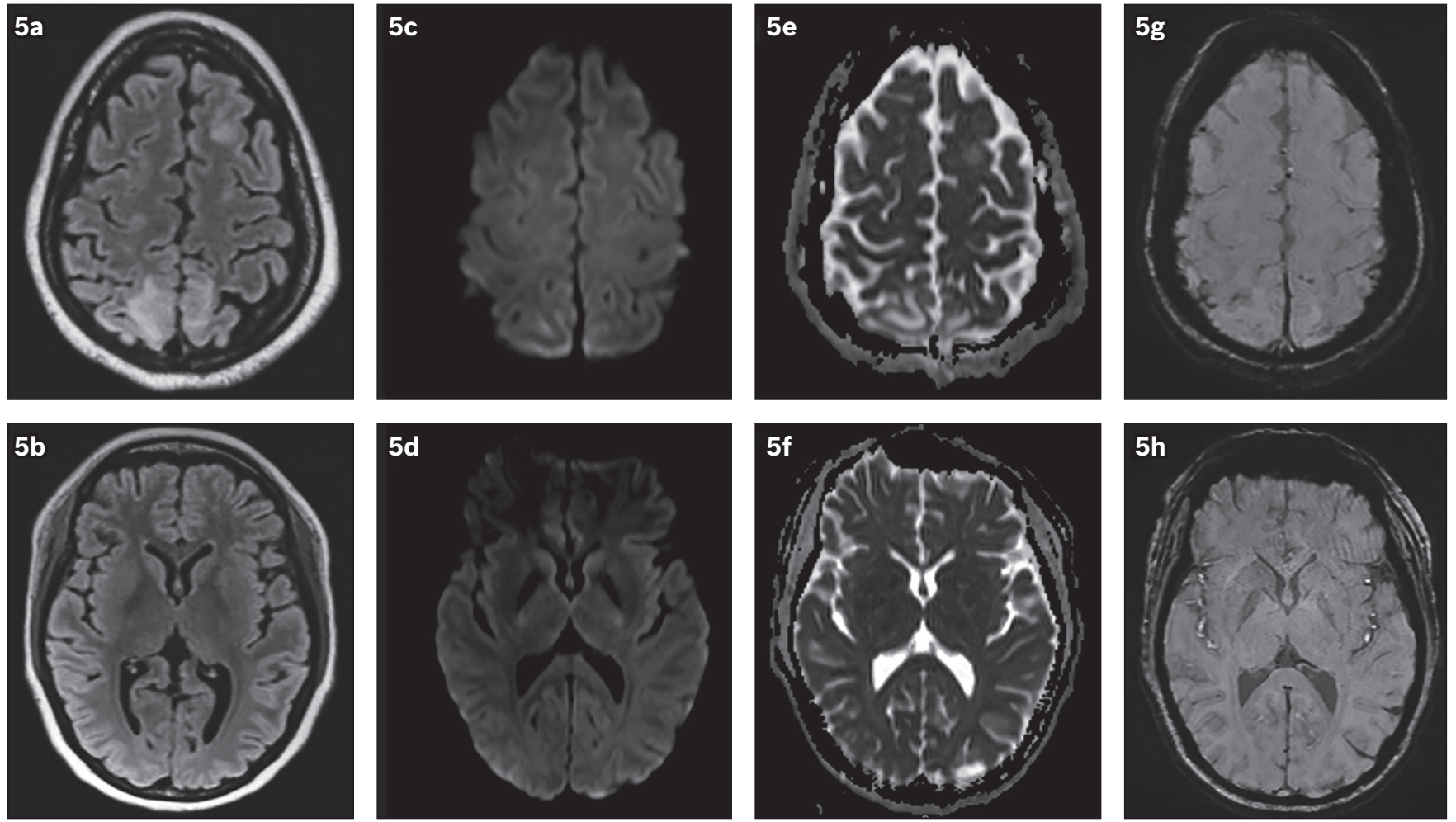

Fig. 5 Posterior reversible encephalopathy syndrome in a 26-year-old woman who presented with seizures and neurodeficits. MR images of the brain show bilateral fairly symmetrical signal abnormalities in the left frontal and bilateral parietal lobes in the cortical and subcortical regions on (a \& b) FLAIR and (c \& d) DW images, with (e \& f) no apparent restricted diffusion in the affected areas and ( $\&$ h) no abnormal susceptibility suggesting intracranial haemorrhage. Normal appearance of the basal ganglia is seen in (b).
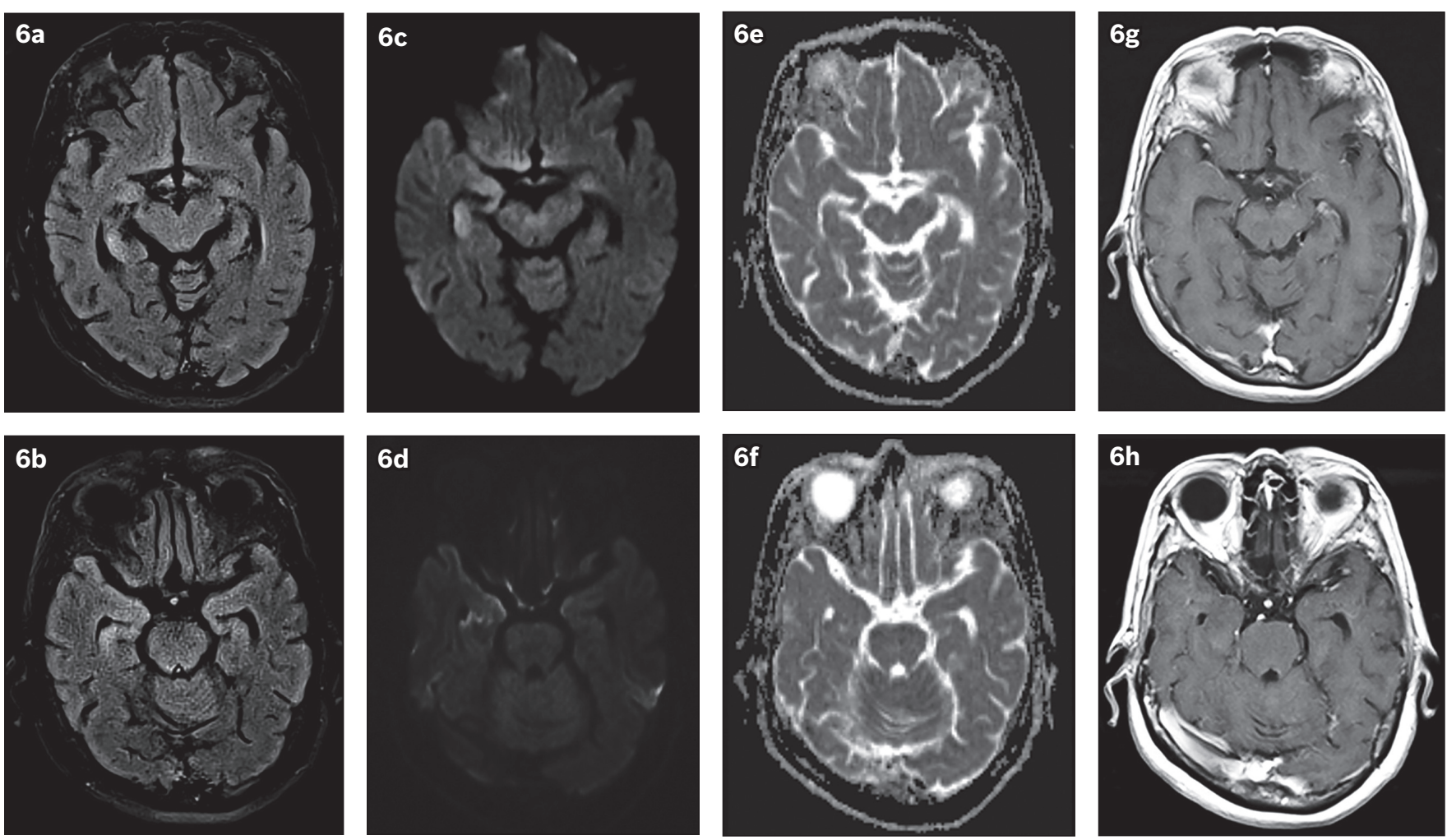

Fig. 6 Postictal state in a 67-year-old woman with a known history of gastrointestinal stromal tumours on imatinib, who presented with seizure. (a \& b) FLAIR and (c \& d) DW images show increased signal intensity and swelling in the body and tail of the right hippocampus. (e \& f) ADC map shows no restricted diffusion detected. The left hippocampus appears unremarkable. ( $\mathrm{g} \& \mathrm{~h}$ ) Post-gadolinium T1-W images show no appreciable abnormal parenchymal or leptomeningeal enhancement. 

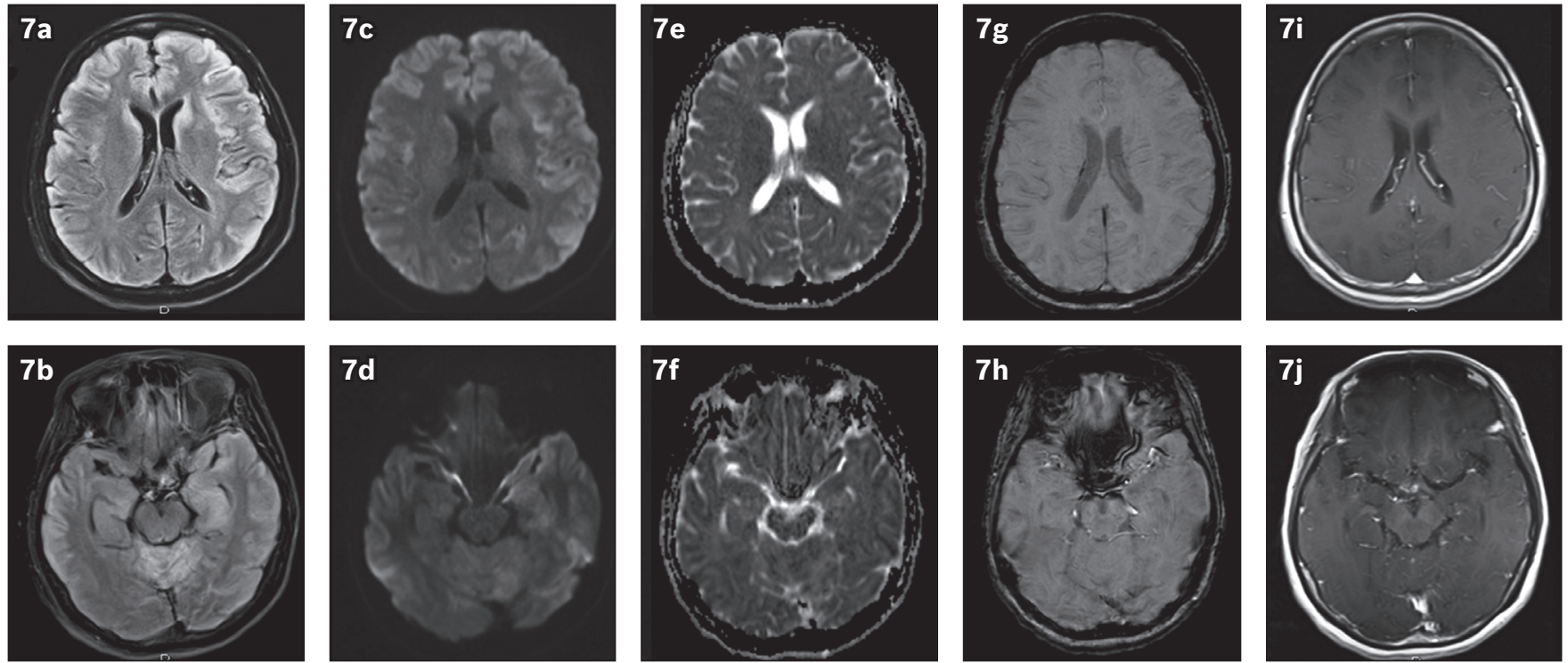

Fig. 7 Voltage-gated potassium channel antibody disease in a 52-year-old woman with suspected encephalitis, who presented with lower limb incoordination and progressive confusion. MR images show ( $\&$ b) areas of FLAIR hyperintensity in the bilateral cortices, left hippocampus, bilateral caudate nuclei and cerebellar hemispheres, with (c-f) associated mild diffusion restriction but without ( $\mathrm{g} \& \mathrm{~h}$ ) abnormal susceptibility or (i \& j) enhancement.

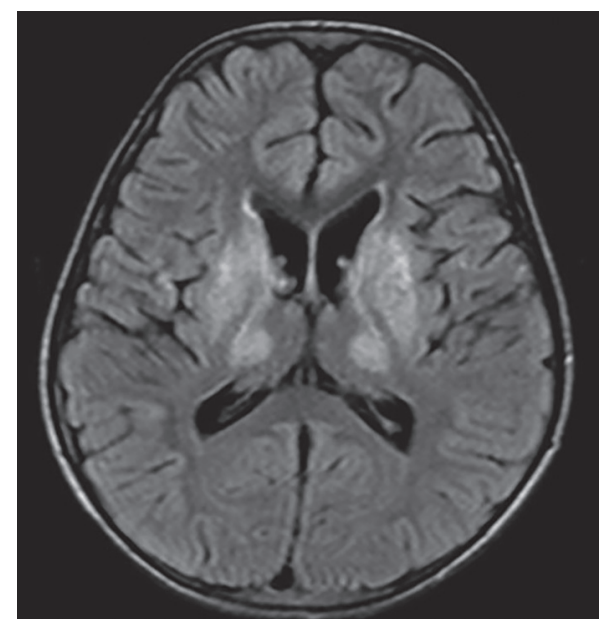

Fig. 8 Wilson's disease in a 15-year-old boy with dysarthria, dystonia and an abnormal liver function test. MR image of the brain shows symmetric abnormal FLAIR hyperintensity in the bilateral basal ganglia and thalami. No cortical signal abnormality is noted.

(Fig. 7). ${ }^{(15)}$ There is increased propensity of development of mesial temporal sclerosis in the chronic stage.

Wilson's disease is an acquired hepatolenticular degeneration due to a disorder of copper metabolism and resultant abnormal accumulation of copper in the liver, brain and eyes. The classic MR features are hyperintensity in the basal ganglia, ventrolateral thalamus, midbrain and pons on T2/FLAIR images (Fig. 8). ${ }^{(16)}$ Diffusion restriction is often present in pre-symptomatic patients in the early stages of disease. In contrast to sCJD, Wilson's disease less commonly involves the cortex and usually presents at an early age with pertinent laboratory results.

Wernicke's encephalopathy is an acute neurological disorder resulting from thiamine (vitamin B1) deficiency, often characterised by the classic triad of oculomotor symptoms, ataxia and altered mental status. This triad can be seen in both alcoholic and non-alcoholic Wernicke's subtypes. MR imaging typically shows bilateral symmetric signal alterations in the medial thalami, periventricular regions of the third ventricle, mammillary bodies, tectal plate and periaqueductal area (Fig. 9). ${ }^{(17)}$ In alcoholic patients, enhancement can be seen in the thalami and mammillary bodies. Although the involvement of medial thalami may mimic the 'double hockey stick' sign, Wernicke's encephalopathy can be differentiated from CJD by its typical MR imaging pattern with relative sparing of the cerebral cortex, and this may be further supported by relevant nutritional history and biochemical test results.

In conclusion, based on clinical features alone, it may be challenging to diagnose $\mathrm{SCJD}$ in vivo because of its wide spectra of clinical presentations. However, paraclinical biomarkers, and DW imaging in particular, play an integral part in the diagnostic workup of sCJD. Careful interpretation of MR images and recognition of the classic features of SCJD not only facilitate a probable diagnosis but also aid in excluding other mimicking disorders.

ABSTRACT A 68-year-old man presented with a three-week history of rapidly progressive dementia, gait ataxia and myoclonus. Subsequent electroencephalography showed periodic sharp wave complexes, and cerebrospinal fluid assay revealed the presence of a 14-3-3 protein. A probable diagnosis of sporadic Creutzfeldt-Jakob disease was made, which was further supported by magnetic resonance (MR) imaging of the brain showing asymmetric signal abnormality in the cerebral cortices and basal ganglia. The aetiology, clinical features, diagnostic criteria, various MR imaging patterns and radiologic differential diagnosis of sporadic Creutzfeldt-Jakob disease are discussed in this article.

Keywords: Creutzfeldt-Jakob disease, human prion disease, MR imaging 

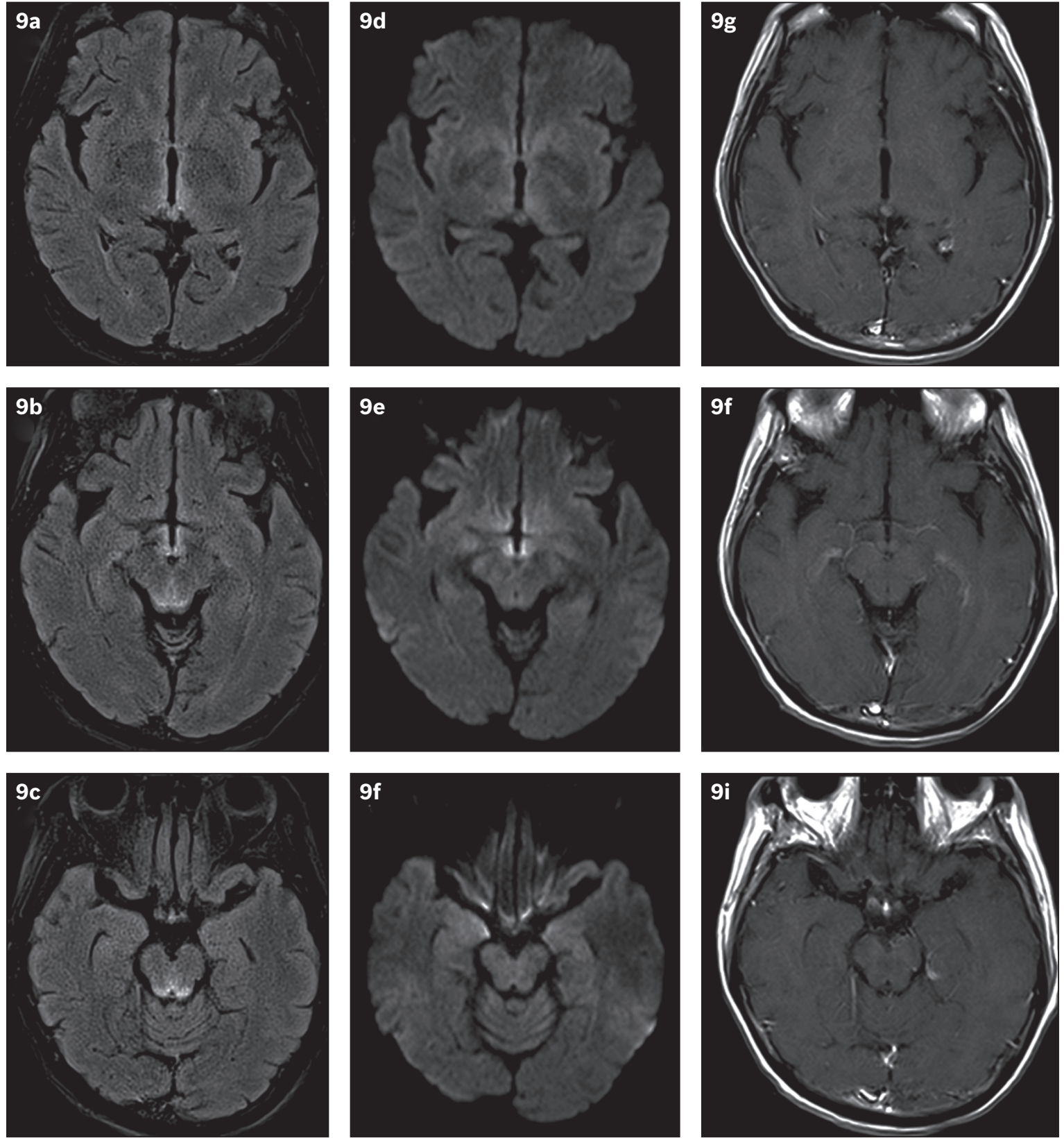

Fig. 9 Probable Wernicke's encephalopathy in a 59-year-old non-alcoholic female patient with gait disturbance, cognitive impairment and memory loss. $(a-c)$ FLAIR and $(d-f)$ DW images of the brain reveal symmetric hyperintensities in the periventricular region of the third ventricle, medial thalami, tectal plate and periaqueductal region, with no $(\mathrm{g}-\mathrm{i})$ associated enhancement. The lack of a history of chronic alcohol abuse suggests that this is a non-alcoholic subtype of Wernicke's encephalopathy.

\section{REFERENCES}

1. Prusiner SB. Prions. Proc Natl Acad Sci U S A 1998; 95:13363-83.

2. Fragoso DC, Gonçalves Filho AL, Pacheco FT, et al. Imaging of Creutzfeldt-Jakob disease: imaging patterns and their differential diagnosis. Radiographics 2017; 37:234-57.

3. World Health Organization. Global surveillance, diagnosis and therapy of human transmissible spongiform encephalopathies: report of a WHO consultation. Geneva, Switzerland, 9-11 February 1998. Available at: http:// www.who.int/csr/resources/publications/bse/WHO_EMC_ZDI_98_9/en/. Accessed October 3, 2018.

4. Zerr I, Kallenberg K, Summers DM, et al. Updated clinical diagnostic criteria for sporadic Creutzfeldt-Jakob disease. Brain 2009; 132(Pt 10):2659-68.

5. Pocchiari M, Puopolo M, Croes EA, et al. Predictors of survival in sporadic Creutzfeldt-Jakob disease and other human transmissible spongiform encephalopathies. Brain 2004; 127(Pt 10):2348-59.

6. Puoti G, Bizzi A, Forloni G, et al. Sporadic human prion diseases: molecular insights and diagnosis. Lancet Neurol 2012; 11:618-28.

7. Mittal S, Farmer P, Kalina P, Kingsley PB, Halperin J. Correlation of diffusionweighted magnetic resonance imaging with neuropathology in Creutzfeldt-Jakob disease. Arch Neurol 2002; 59:128-34.

8. Caobelli F, Cobelli M, Pizzocaro C, et al. The role of neuroimaging in evaluating patients affected by Creutzfeldt-Jakob disease: a systematic review of the literature. J Neuroimaging 2015; 25:2-13.
9. Huang BY, Castillo M. Hypoxic-ischemic brain injury: imaging findings from birth to adulthood. Radiographics 2008; 28:417-39; quiz 617.

10. Ren S, Chen Z, Liu M, Wang Z. The radiological findings of hypoglycemic encephalopathy: a case report with high b value DWI analysis. Medicine (Baltimore) 2017; 96:e8425.

11. Soares BP, Provenzale JM. Imaging of herpesvirus infections of the CNS. AJR Am J Roentgenol 2016; 206:39-48.

12. Bartynski WS. Posterior reversible encephalopathy syndrome, part 2: controversies surrounding pathophysiology of vasogenic edema. AJNR Am j Neuroradiol 2008; 29:1043-9.

13. Bartynski WS. Posterior reversible encephalopathy syndrome, part 1 : fundamental imaging and clinical features. AJNR Am J Neuroradiol 2008; 29:1036-42.

14. Kim JA, Chung JI, Yoon PH, et al. Transient MR signal changes in patients with generalized tonicoclonic seizure or status epilepticus: periictal diffusionweighted imaging. AJNR Am J Neuroradiol 2001; 22:1149-60.

15. Kotsenas AL, Watson RE, Pittock SJ, et al. MRI findings in autoimmune voltagegated potassium channel complex encephalitis with seizures: one potential etiology for mesial temporal sclerosis. AJNR Am J Neuroradiol 2014; 35:84-9.

16. Kim TJ, Kim IO, Kim WS, et al. MR imaging of the brain in Wilson disease of childhood: findings before and after treatment with clinical correlation. AJNR Am I Neuroradiol 2006; 27:1373-8.

17. Manzo G, De Gennaro A, Cozzolino A, et al. MR imaging findings in alcoholic and nonalcoholic acute Wernicke's encephalopathy: a review. Biomed Res Int $2014 ; 2014: 503596$. 


\section{SINGAPORE MEDICAL COUNCIL CATEGORY 3B CME PROGRAMME} (Code SMJ 201812B)

Question 1. Regarding imaging techniques supporting the diagnosis of Creutzfeldt-Jakob disease (CJD), which is highly sensitive and specific, and currently widely in use?

(a) Dynamic susceptibility contrast magnetic resonance (MR) imaging.

(b) Diffusion-weighted MR imaging.

(c) Positron emission tomography/computed tomography.

(d) Susceptibility-weighted MR imaging.

Question 2. Common MR imaging patterns of sporadic CJD include:

(a) Bilateral symmetric T2 hyperintense signal abnormalities in the mesial temporal lobes and hippocampi.

(b) Diffuse T2 hyperintensities in the subcortical and deep white matter of the parietal and occipital lobes, with or without microhaemorrhages.

(c) Asymmetric areas of restricted diffusion in the cerebral cortex and/or basal ganglia regions.

(d) Symmetric restricted diffusion in the dorsomedial thalami.

Question 3. A probable CJD is diagnosed based on:

(a) Detection of scrapie prion protein on biopsy.

(b) Positive electroencephalography (EEG) and MR imaging findings on a clinical background of rapidly progressive dementia and myoclonus.

(c) Equivocal MR imaging and EEG results, with the presence of 14-3-3 protein in cerebrospinal fluid (CSF) in a patient without dementia.

(d) Rapidly progressive dementia in less than two years with pyramidal signs but negative MR imaging, CSF analysis and EEG results.

Question 4. Regarding CJD:

(a) It is caused by abnormal formation and accumulation of scrapie prion protein in brain tissue.

(b) There are several subtypes and variant CJD is the best understood subtype thus far.

(c) It is rapidly progressive in clinical course and invariably fatal regardless of treatment.

(d) Visual disturbance is an uncommon clinical presentation.

Question 5. Regarding the differential diagnosis of CJD:

(a) MR imaging is the key to differentiate it from hypoglycaemic encephalopathy.

(b) Encephalitis usually spares the limbic system, unlike CJD.

(c) In a postictal state, transient signal changes can usually be seen in the hippocampi and corpus callosum

(d) Wernicke's encephalopathy can also be seen in non-alcoholic individuals.

True

False

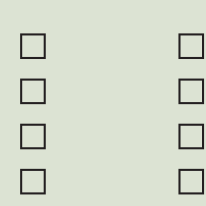

$\square$

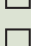

$\square$
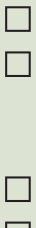

$\square$

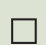

$\square$

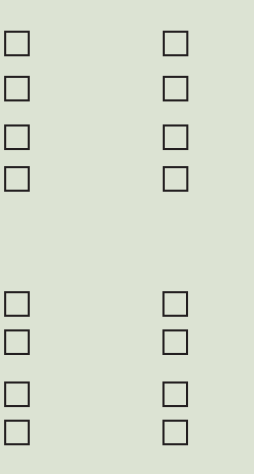

\section{Doctor's particulars:}

Name in full:

MCR no.:

Specialty:

Email:

\footnotetext{
SUBMISSION INSTRUCTIONS:

Visit the SMJ website: http://www.smj.org.sg/current-issue and select the appropriate quiz. You will be redirected to the SMA login page.

For SMA member: (1) Log in with your username and password (if you do not know your password, please click on 'Forgot your password?'). (2) Select your answers for each quiz and click 'Submit'.

For non-SMA member: (1) Create an SMJ CME account, or log in with your SMJ CME username and password (for returning users). (2) Make payment of SGD 21.40 (inclusive of $7 \%$ GST) via PayPal to access this month's quizzes. (3) Select your answers for each quiz and click 'Submit'.

RESULTS:

(1) Answers will be published online in the SMJ February 2019 issue. (2) The MCR numbers of successful candidates will be posted online at the SMJ website by 1 February 2019. (3) Passing mark is $60 \%$. No mark will be deducted for incorrect answers. (4) The SMJ editorial office will submit the list of successful candidates to the Singapore Medical Council. (5) One CME point is awarded for successful candidates. (6) SMC credits CME points according to the month of publication of the CME article (i.e. points awarded for a quiz published in the December 2017 issue will be credited for the month of December 2017, even if the deadline is in January 2018)

Deadline for submission (December 2018 SMJ 3B CME programme): 12 noon, 25 January 2019.
} 